\title{
A pharmacokinetic-pharmacodynamic model for the MET-TKI, savolitinib, to explore target inhibition requirements for anti-tumour activity
}

\author{
Rhys Jones ${ }^{1}$, Mike Grondine ${ }^{2}$, Alexandra Borodovsky², Maryann San Martin ${ }^{2}$, Michelle \\ DuPont $^{2}$, Celina D'Cruz ${ }^{2}$, Alwin Schuller ${ }^{2}$, Ryan Henry ${ }^{2}$, Evan Barry ${ }^{2}$, Lillian Castriotta ${ }^{2}$, \\ Rana Anjum ${ }^{2}$, Klas Petersson ${ }^{3}$, Tarjinder Sahota ${ }^{2}$, and Ghada Ahmed ${ }^{2}$ \\ ${ }^{1}$ AstraZeneca PLC \\ ${ }^{2}$ AstraZeneca \\ ${ }^{3}$ qPharmetra LLC
}

May 5, 2020

\begin{abstract}
Background and purpose: Savolitinib (AZD6094, HMPL-504, volitinib) is an oral, potent, and highly selective MET receptor tyrosine kinase inhibitor. This series of studies aimed to develop a pharmacokinetic-pharmacodynamic (PK/PD) model to link inhibition of MET phosphorylation (pMET) by savolitinib with anti-tumour activity. Experimental approach: Cell line-derived xenograft (CDX) experiments using human lung cancer (EBC-1) and gastric cancer (MKN-45) cells were conducted in athymic nude mice using a variety of doses and schedules of savolitinib. Tumour pMET changes and growth inhibition were calculated after 28 days. Population PK/PD techniques were used to construct a PK/PD model for savolitinib. Key results: Savolitinib showed dose- and schedule-dependent anti-tumour activity in the CDX models, with more frequent, lower dosing schedules (e.g. twice daily) being more effective than intermittent, higher dosing schedules (e.g. 4 days on $/ 3$ days off or 2 days on/5 days off). There was a clear exposure-response relationship, with maximal suppression of pMET of $>90 \%$. Data from additional CDX and patient-derived xenograft (PDX) models overlapped, allowing the calculation of a single EC50 of $0.38 \mathrm{ng} / \mathrm{mL}$. Tumour growth modelling demonstrated that prolonged, high levels of pMET inhibition $(>90 \%)$ were required for tumour stasis and regression in the models. Conclusion and implications: High and durable levels of MET inhibition by savolitinib are needed for optimal monotherapy anti-tumour activity in preclinical models. The modelling framework developed here can be used to translate tumour growth inhibition from the mouse to human, and thus guide choice of clinical dose and schedule.
\end{abstract}

\section{Introduction}

MET (mesenchymal epithelial transition factor) is a receptor tyrosine kinase (RTK) associated with the regulation of tissue repair, proliferation, and survival in normal physiology (Matsumoto \& Nakamura, 1996). Dysregulation of MET signalling mediates mitogenesis, angiogenesis and invasion and has been implicated in a number of malignancies including papillary renal cell carcinoma (PRCC), gastric and lung cancers (Albiges et al., 2014; Awad et al., 2016; Soman, Correa, Ruiz, \& Wogan, 1991), Aberrant MET signalling occurs through a number of mechanisms including overexpression of MET or its ligand HGF (hepatocyte growth factor), MET gene amplification, MET gene mutation/ rearrangement and changes in ligand-induced autocrine or paracrine signalling (Raghav et al., 2018). Furthermore, MET gene amplification could be detected in about $5-22 \%$ of lung adenocarcinomas patients developing acquired resistance to first generation epidermal growth factor receptor tyrosine kinase inhibitor (EGFR TKI) (Arcila et al., 2011; Bean et al., 2007; Engelman et al., 2007; Sequist et al., 2011). Therefore MET has been a target of significant clinical 
interest and drug discovery efforts and several strategies are being developed to therapeutically target MET, including small molecule kinase inhibitors. Savolitinib (AZD6094, HMPL-504, volitinib) is an oral, potent, and highly selective MET TKI (Gavine et al., 2015; Jia et al., 2014).

A pharmacokinetic-pharmacodynamic (PK/PD) model relating savolitinib exposure to inhibition of MET phosphorylation (pMET) and anti-tumour activity in the Hs746T gastric model was recently published (Gu et al., 2019). This model estimated that as little as $10 \%$ continuous inhibition of pMET is required to prevent the tumour growing (stasis), which equates to plasma concentration of $1.1 \mathrm{ng} / \mathrm{ml}$. Work describing a PK/PD model for crizotinib, an ALK/ROS1 inhibitor with MET-inhibitory activity, has also been previously published, linking drug exposure to inhibition of pMET (Yamazaki et al., 2008). The model was built on data generated in athymic mice implanted with GTL16 gastric carcinoma cells and U87MG glioblastoma cells; it indicated that $>90 \%$ inhibition of pMET is required to drive significant anti-tumour activity, with durable inhibition desirable for optimal anti-tumour activity. Another group investigated two MET inhibitors in preclinical models, assessing PK, pMET inhibition in tumour samples, and anti-tumour activity (Bladt et al., 2013). The two compounds were primarily distinguished by differences in their plasma half-life, with more durable inhibition of pMET observed for the compound with a longer half-life, and consequently greater anti-tumour activity. The compound with the longer half-life was taken into the clinic as MSC2156119J (tepotinib, EMD-1214063), and tested in a Phase I trial to assess PK, inhibition of pMET, and anti-tumour activity (Falchook et al., 2014). Subsequently, the recommended Phase II dose was set based on achieving a plasma exposure at steady state that delivers $>90 \%$ inhibition of pMET in the tumour over the dosing interval (Falchook et al., 2014).

Thus, it seems that potent $(>90 \%)$ and durable inhibition of pMET is desirable to maximise anti-tumour activity. However, the data from Gu et al., (2019) for savolitinib suggests a lower threshold, but that analysis focussed on a single in vivo model. It is important to confirm the requirements for savolitinib, and, since savolitinib is being investigated for the treatment of multiple tumour types in the clinic, an important question to address is whether the same relationships are apparent across tumour models. Mathematical modelling is a useful tool to integrate a number of datasets and quantitatively address this question; such a model can then be used to predict the likely time-course of pMET inhibition for a given dose and schedule being tested in the clinic.

The current investigation analysed pMET inhibition and anti-tumour activity in gastric (MKN-45) and non-small cell lung cancer (NSCLC; EBC-1) cell line xenograft models using several doses and schedules of savolitinib. Savolitinib has also been tested in a number of additional cell line-derived xenograft (CDX) and patient-derived xenograft (PDX) models carrying MET copy number gain or amplification (Schuller et al., 2015) (Gavine et al., 2015) (Henry et al., 2016). These additional datasets along with the EBC-1 and MKN-45 models presented here were used to develop a mathematical model relating savolitinib exposure and pMET inhibition to anti-tumour activity. The mathematical model was used to determine the extent and duration of pMET inhibition needed for optimal efficacy and to address the question of whether these requirements differ across the CDX and PDX models tested.

\section{Methods}

\section{Animals}

Female athymic nude mice were purchased from Charles River Laboratories (Wilmington, MA). Mice were housed under pathogen-free conditions in individual ventilated cages at our Association for the Assessment and Accreditation of Laboratory Animal Care accredited facility in Waltham, MA. All animal manipulations were conducted in a biosafety cabinet maintained under positive pressure. Mice were $5-6$ weeks old at the time of tumour implantation. All animal studies were conducted in accordance with the guidelines established by the internal Institutional Animal Care and Use Committee and reported following the Animal Research: Reporting In Vivo Experiments guidelines (Kilkenny, Browne, Cuthill, Emerson, \& Altman, 2010). 


\section{Xenograft anti-tumour activity studies}

Five million EBC-1 human lung cancer or ten million MKN-45 human gastric cancer cells were injected subcutaneously in the right flank of athymic nude mice in a volume of $0.1 \mathrm{~mL}$ containing $50 \%$ matrigel. Mice were randomised based on tumour volumes using stratified sampling, and enrolled into control and treatment groups. Oral dosing began when mean tumour size reached $\sim 175 \mathrm{~mm}^{3}$ for EBC-1 and $\sim 250 \mathrm{~mm}^{3}$ for MKN-45. In order to determine the anti-tumour activity of savolitinib in theMET -amplified gastric cancer xenograft model (MKN-45), we treated mice daily (QD), with dose levels ranging from $2.5-25 \mathrm{mg} / \mathrm{kg}$, and twice a day (BID; second dose given 8 hours after the first), with dose levels in the range 1.25-12.5 $\mathrm{mg} / \mathrm{kg}$ (Supplementary Table S1). Doses and schedules were selected to deliver a broad dose range overall and encompass clinical exposures observed, and the BID groups had a matching QD group receiving the same daily total dose. In the EBC-1 model, a more extensive range of doses and dose schedules were tested including QD, BID and intermittent schedules. The discontinuous schedules explored included 2 days on 5 days off $(2 / 5), 4$ days on 3 days off (4/3), or every other day (Q2D). Savolitinib was dosed alone or in combination with a pan-CYP inhibitor (100 $\mathrm{mg} / \mathrm{kg}$ intraperitoneal, 1 hour prior to savolitinib dosing), which prolonged savolitinib PK half-life by reducing the elimination rate so that the plasma concentration-time profile better matches that seen in the clinic (1-aminobenzotriazole; ABT Sigma catalog number: A3940250mg, Lot number: 087M4078V).

Tumour volumes, body weight, and tumour condition were recorded at least twice weekly for the duration of the study. The tumour volume was calculated (taking length to be the longest diameter across the tumour and width to be the corresponding perpendicular diameter) using the formula (equation 1):

[1] $T V=$ length $\times$ width $^{2} \times 0.52$

Where TV is tumour volume, length in $\mathrm{mm}$, width in $\mathrm{mm}$.

Growth inhibition from the start of treatment was assessed by comparison of the differences in tumour volume between control and treated groups according to the following equations for percentage inhibition, and percentage regression (equation 2 and 3 ).

$$
\begin{aligned}
& {[2] \% \text { inh }=100 \times\left(\frac{\mathrm{TV}_{\text {cont }}-\mathrm{TV}_{\text {treat }}}{\mathrm{TV}_{\text {cont }}-1}\right)} \\
& {[3] \% \text { reg }=100 \times\left(1-\mathrm{TV}_{\text {treat }}\right)}
\end{aligned}
$$

Where\% inh is the calculated percentage of tumour growth inhibition, $\%$ reg is the calculated percentage of tumour growth shrinkage, $T V_{\text {cont }}$ is the geometric mean tumour volume of the control group, $T V_{\text {treat }}$ is the geometric mean tumour volume of the treated group

Savolitinib has been investigated in a number of additional CDX and PDX models. These have been combined with MKN-45 and EBC-1 datasets, presented here as part of the analysis (Supplementary Table S2).

\section{Mouse pharmacokinetics and pharmacodynamics assessments}

Whole blood was collected under xylazine-ketamine anaesthesia by cardiac puncture and plasma frozen at $-80^{\circ} \mathrm{C}$ until analysis. PK samples were collected at multiple time points both after single (first) dose administration and at the end of study (repeat dose administration). Plasma samples were analysed for parent concentration using a protein precipitation extraction procedure followed by LC-MS/MS detection; analysed and processed using MassLynx and TargetLynx, respectively (Waters, Milford, MA, USA).

For assessment of target engagement, tumours were excised and frozen at $-80^{\circ} \mathrm{C}$ until the time of analysis. Analysis was conducted by Western blot (WB).

Tumour fragments were homogenised in lysis buffer. Proteins, separated by SDS-PAGE, were transferred to nitrocellulose membranes, and incubated with the following antibodies (all at 1:1000 in TBST BSA 3\%): pMET (Y1234/1235) Cell Signaling Technology (CST) 3077, MET CST 8198, followed by incubation with 
a secondary horseradish peroxidase-conjugated antibody and chemiluminescence detection. The lower limit of quantification for pMET was set to $1 \%$ of cell-specific baseline pMET value.

\section{Pharmacokinetic-pharmacodynamic analysis and software}

Population PK/PD analysis was carried out using nonlinear mixed effects modelling as implemented in NONMEM 7.3 (ICON Development Solutions, Elicott City, MD, USA) and the GNU GFortran compiler (Versions 4.6.0, [ftp://ftp.globomaxnm.com/Public/nonmem7/compilers]). The first-order conditional estimation with $\eta-\varepsilon$ interaction (FOCE-I) was used. Model selection was based on the change in NONMEM objective function value (OFV; for nested models), Aikaiki information criteria (for non-nested models), and visual improvements in the diagnostic plots. For nested models, decreases in the OFV of at least 6.63 $(\mathrm{p}<0.01$; degrees of freedom $=1)$ and $10.83(\mathrm{x} 2, \mathrm{p}<0.001$; degrees of freedom $=1)$ were used as cut-off values for forward inclusion and backward elimination, respectively. Post-processing of NONMEM analysis results was carried out in $\mathrm{R}$ version 3.0.2 (Comprehensive R Network, http://cran.r-project.org). The precision of the parameter estimates was evaluated throughout the model development by examining the asymptotic standard errors calculated using the covariance routine in NONMEM and, for final models, using a bootstrap analysis was carried out using Perl-speaks-NONMEM, versions 3.5.3 and 4.2.0 (Lindbom, Pihlgren, \& Jonsson, 2005).

\section{Population pharmacokinetic model}

Data from all CDX and PDX experiments were pooled together for population PK analysis. Both one- and two-compartment PK models with first-order absorption and elimination were initially tested to fit the mouse plasma savolitinib PK. The exposure-dependent clearance was modelled via Michaelis-Menten kinetics, and absorption was captured by a dose-dependent relative bioavailability. Furthermore, the effect of ABT was incorporated on maximum elimination $\left(\mathrm{V}_{\max }\right)$ to account for additional reduction in elimination rate as an estimated change in $\mathrm{V}_{\max }$ conditioned on the categorical variable of ABT present (yes/no). Slight variations in exposure for some studies were accounted for by estimating separate bioavailability for these studies.

\section{Exposure-pMET model}

Exploratory analyses of savolitinib concentration versus percentage inhibition from baseline in pMET (as a marker of target suppression) showed a concentration-dependent response. Plotting the data in this way also showed a lack of hysteresis and therefore no or minimal time delay between plasma concentration and change in pMET. As a result, a direct response model linking the time course of plasma concentration to the time course of phosphorylated MET was developed applying plasma concentration in the central PK compartment as driving force (Supplementary Figure S1). The concentration-pMET relationship was fitted to a Hill equation (equation 4 ):

$$
\text { [4] } p M E T=\text { base }_{\text {pMET }}\left(1-\frac{E_{\max } C_{p} \gamma}{\mathrm{EC}_{50}{ }^{\gamma}+C_{p} \gamma}\right)
$$

where base pMET $_{\text {is }}$ the baseline tumour pMET level, $C_{p}$ is the savolitinib plasma concentration, $E_{\max }$ is the maximum suppression at infinite savolitinib concentration, $\mathrm{EC}_{50}$ is the concentration at which $50 \%$ of the maximum suppression is achieved, and $\gamma$ is the Hill factor. Covariate testing included cell line type on $\mathrm{EC}_{50}$ and/or $\mathrm{E}_{\max }$ (if range of data allowed estimation of a separate $\mathrm{E}_{\max }$ ) and method of detection (WB vs ELISA) as categorical variables.

\section{pMET tumour growth inhibition model}

The relationship between pMET and tumour growth inhibition (TGI) was sequentially modelled keeping concentration-pMET relationship fixed. The Simeoni model was used as the basis of tumour dynamic modelling (Simeoni et al., 2004). The percent pMET suppression was first transformed from $0-1$ to 0 [?] scale (equation 5) to allow for stable estimation and assumed to be the sole driver of tumour growth inhibition: 
[5] $\operatorname{pMET}_{T}=\left(\frac{\mathrm{pMET}}{1-p M E T}\right)$

Linear, power-linear (equation 6) and $\mathrm{E}_{\max }$ (equation 7) pMET-tumour kill relationships were explored as following:

$$
\begin{aligned}
& \text { [6] } \quad \text { Effect }=\left(\text { slope } \bullet \mathrm{pMET}_{T}\right)^{\gamma} \\
& \text { [7] } \quad \text { Effect }=E_{\max } \frac{\mathrm{pMET}_{T}}{\left(\mathrm{pMET}_{T}+\mathrm{pMET}_{50}\right)}
\end{aligned}
$$

where $\mathrm{pMET}_{50}$ is the transformed pMET supression required for $50 \%$ maximal effect. Covariate testing explored the possibility of cell line specific pMET-tumour kill parameters. Supplementary Figure S1 shows a schematic of the mathematical model used to link savolitinib plasma concentration to MET phosphorylation that drives anti-tumour activity.

\section{Model diagnostics qualification}

Diagnostic plots of observed data vs population and individual predictions were examined for overall fit and lack of bias in the predicted values. Plots of conditional weighted residuals and between-subject variability were inspected for evidence of systematic lack of fit, and to confirm the absence of bias in the error distributions. The final model was determined on the basis of maximised likelihood (lowest OFV), physiological plausibility of parameter values, and successful numerical convergence. Model parameter values should preferably have a standard error of estimation no higher than $50 \%$ of the parameter estimate, and there should not be significant bias in the random effects estimates.

To verify that residuals were randomly distributed around zero, and that no significant structural bias remained, individual weighted residuals and conditional weighted residuals were plotted versus the population predictions and versus time, stratified by dose and/or study. A normal quantile plot and a density plot of conditional weighted residuals were used to validate approximate normal error distribution with a mean of approximately zero.

\section{Results}

\section{Pharmacokinetic properties and population PK model for savolitinib in mice}

Mouse PK data from the two main studies in the MKN-45 and EBC-1 models alongside PK data from other studies (listed in Supplementary Table S1) have been used to define the mouse population PK model. It has been shown previously that savolitinib has a biphasic profile following intravenous administration and an elimination half-life of $1.5 \mathrm{~h}$ (Gu et al., 2013), and these data were used alongside the oral data (described below) to estimate the PK model parameters. Following oral dosing, savolitinib was rapidly absorbed, with maximal concentrations occurring between 1-2 h. A dose-proportional increase in exposure was observed up to $30 \mathrm{mg} / \mathrm{kg}$ and, above $30 \mathrm{mg} / \mathrm{kg}$, there was a greater than proportional increase in exposure with dose, and a lengthening of the elimination half-life. This observation was consistent with saturation of a clearance mechanism. The co-dosing of cytochrome P450 inhibitor ABT at $100 \mathrm{mg} / \mathrm{kg}$ in the EBC- 1 study also prolonged the half-life of savolitinib to $3.5 \mathrm{~h}$, which was more consistent with that seen in patients $(3$ to $6 \mathrm{~h}$ ); a comparison of the simulated mouse PK profile with and without ABT is shown in Supplementary Figure S2A. The structural PK model was two-compartment with first order absorption and MichaelisMenten elimination applied ( $\Delta \mathrm{OFV}=15.8, \mathrm{x} 2 \mathrm{p}<0.01$ compared to one-compartment model). Accounting for effect of $\mathrm{ABT}$ on $\mathrm{V}_{\max }$ rather than on $\mathrm{K}_{\mathrm{m}}$ led to a drop of 3.6 in OFV (same number of parameters).

Final mouse PK model parameters are shown in Table 1. Comparisons of model simulations against representative observed data from the EBC-1 and MKN-45 studies are shown in Supplementary Figure S2B-D. Overall, the model adequately described the plasma concentration-time profiles observed across dose levels and studies investigated; goodness of fit plots are shown in Supplementary Figure S3.

\section{Savolitinib exposure-pMET model in mouse}


Across the CDX and PDX models investigated, after a single dose of savolitinib, pMET inhibition responded to savolitinib plasma concentrations with rapid onset and no apparent delay, as evidenced by lack of a hysteresis loop when plotting savolitinib exposure against inhibition of pMET (Figure 1). The observed maximum inhibition of pMET coincided with savolitinib $t_{\max }$ of $1-2 \mathrm{~h}$; recovery of pMET to baseline continued to track with the PK profile. We concluded that no delay between plasma PK and pMET inhibition was evident, and consideration for an indirect-response model would not be required. A sigmoidal $\mathrm{E}_{\max }$ model provided adequate description of the concentration-pMET relationship. The western blots for pMET and total MET protein measured in the EBC-1 and MKN-45 studies presented here are shown in Supplementary Figure S4. Within the dataset used in this exercise, the PD response following multiple days of dosing was investigated across several studies and shown to mirror that seen following a single dose. Therefore, it was assumed that no change in inhibition of pMET over time is observed and all data (single dose and repeat dose) are plotted in Figure 1. There was no statistically-significant difference between cell lines in $\mathrm{EC}_{50}$ or $\mathrm{E}_{\max }$ and single estimates of $0.38 \mathrm{ng} / \mathrm{mL}$ (95\% CI 0.25-0.5) and 97\% (95\% CI 94-97) adequately described exposure-pMET relationship across all cell lines. For some previously published studies used in this analysis, changes in pMET were measured using Meso Scale Discovery ${ }^{\circledR}$ (MSD). A comparison was made between datasets generated by MSD and WB and differences in the exposure-response parameters between the methods were not statistically significantly different. For both methods, $\mathrm{EC}_{50}$ estimates were within approximately 1.5-fold (ELISA $0.38 \mathrm{ng} / \mathrm{ml}$; WB $0.25 \mathrm{ng} / \mathrm{ml}$ ) with overlapping confidence intervals (ELISA 95\% CI 0.27-0.54; WB 95\% CI 0.15-0.42), and a resultant insignificant drop of 6 in OFV. Figure 1 shows the final model overlaid on observed concentration-pMET data from across all datasets used here. Figure 2 shows a comparison between the observed time-course of pMET inhibition measured in the EBC-1 and MKN-45 studies presented here and model simulations. Final model parameters are shown in Supplementary Tables S3 and S4. Goodness of fit plots are shown in Figure S5. The model was used to derive the concentration required for near complete inhibition (90\%) of pMET (EC90 $3.4 \mathrm{ng} / \mathrm{mL})$.

\section{Savolitinib showed dose- and schedule-dependent anti-tumour activity in the EBC-1 NSCLC and MKN-45 gastric cancer xenograft models}

In the EBC-1 model, there was a dose-dependent increase in the level of anti-tumour activity with once-daily doses of $2.5 \mathrm{mg} / \mathrm{kg}$ (77\% TGI), $5 \mathrm{mg} / \mathrm{kg}$ (79\% TGI), $10 \mathrm{mg} / \mathrm{kg}$ (87\% TGI) $30 \mathrm{mg} / \mathrm{kg}$ (76 to $94 \%$ TGI) and $100 \mathrm{mg} / \mathrm{kg}$ (58\% regression) (Supplementary Table S2; Figure 3A). We found that although $30 \mathrm{mg} / \mathrm{kg}$ BID and $100 \mathrm{mg} / \mathrm{kg} \mathrm{2/5}$ both deliver approximately the same AUC over a weekly cycle, the continuous BID dose resulted in greater anti-tumour effects (Supplementary Table S2). Of all schedules tested, the discontinuous schedules showed the least anti-tumour response, with the $100 \mathrm{mg} / \mathrm{kg}$ Q2D or 4/3 resulting in TGIs similar to mice receiving $30 \mathrm{mg} / \mathrm{kg}$ QD or $15 \mathrm{mg} / \mathrm{kg}$ BID, despite receiving twice as much compound on a weekly basis (Supplementary Table S2; Figure 3A). The addition of ABT increased the half-life of savolitinib in the mouse, which increased the duration of pMET inhibition at a given dose level, resulting in improved efficacy compared to the non ABT groups. For example, $2.5 \mathrm{mg} / \mathrm{kg}$ QD delivers $77 \%$ TGI while $2.5 \mathrm{mg} / \mathrm{kg}+\mathrm{ABT}$ delivers $19 \%$ regression; $30 \mathrm{mg} / \mathrm{kg}$ delivers 76 to $94 \%$ TGI whilst $30 \mathrm{mg} / \mathrm{kg}+$ ABT delivers $75 \%$ regression. (Supplementary Table S2; Figure 3B). Similar results of dose- and schedule-dependent anti-tumour activity were observed in the MKN-45 gastric cancer xenograft model (Figure 3C, Supplementary Table S5). Body weight time-course data are shown for both the EBC-1 and MKN-45 studies in Supplementary Figure S6. In the MKN-45 study, some animals were removed mid-study due to tumour ulcerations, but this had no statistical effect on the calculation of tumour growth inhibition (derived from the geometric mean tumour volume). For the PK/PD modelling, each animal was modelled individually and therefore the data were used as is.

\section{Xenograft tumour growth model and savolitinib effect}

Across the datasets presented here (EBC-1 and MKN-45) and those that were previously published and used in this analysis, no delay between start of savolitinib treatment and onset of effect was observed, thus the Simeoni model (equation 5) could be reduced to a single compartment of growing tumour cells (T) (equation 
8), growing with rate constant $\left(\lambda_{0}\right)$. Both exponential and linear growth rates $\left(\lambda_{1}\right.$, linear and $\lambda_{0}$, exp $)$ were tested for each cell line and informed by data from control (vehicle-treated) cohorts (equations 9 and 10, fixed to 20). $\lambda_{Q}$ is the ratio of the growth rates, used in the change point calculation. The TGI data showed that there was a negative correlation between growth and inhibitory effect (i.e. faster growing cells had a higher inhibition rate and vice versa). Therefore, the effect (E) of pMET suppression on tumour inhibition rate was modelled as proportional to the growth rate of the cell line (equation 11). E thus becomes a factor of the intrinsic growth rates of the tumour model rather than an inhibitory rate constant. When $\mathrm{E}=1$, the inhibition equals the growth, and tumour stasis is reached. During the periods where $\mathrm{E}>1$, inhibition is greater than growth and the tumour is shrinking. Incorporating a growth-dependent drug effect into the model has led to a better model fit and significant drop in OFV $\left(\Delta \mathrm{OFV}=461.8, \mathrm{X}^{2} \mathrm{p}<0.0001\right)$.

$$
\begin{aligned}
& \text { [8] } \frac{\mathrm{dT}}{\mathrm{dt}}=G-G \bullet E \\
& \text { [9] } \lambda_{Q}=\frac{\lambda_{0 i} \bullet T}{\lambda_{1}} \\
& \text { [10] } \quad G=T \bullet \lambda_{0 i} \bullet \frac{1}{\left(1+\lambda_{Q}^{\phi}\right)^{\frac{1}{\phi}}} \\
& \text { [11] } E=\left(p M E T_{T} \bullet \text { slope }\right)^{\gamma} \\
& \text { [12] } \lambda_{0 i}=\lambda_{0} \bullet \frac{T^{5}}{\left(T^{5}+T_{\text {frac }}^{5}\right)}+\lambda_{0} \bullet \lambda_{\text {frac }} \bullet\left(1-\frac{T^{5}}{\left(T^{5}+T_{\text {frac }}^{5}\right)}\right)
\end{aligned}
$$

The data did not support estimation of an $\mathrm{E}_{\max }$ relationship for pMET effect (described in equation 11 above). A power-linear function was not worse in model fit than the $\mathrm{E}_{\max }$ model according to Aikaiki information criteria $(\triangle \mathrm{OFV}=-0.25, \mathrm{k}=1)$ (equation 13$)$.

$$
[\mathbf{1 3}] \text { Effect }=\left(\text { slope } \bullet \mathrm{pMET}_{T}\right)^{\gamma}
$$

For the studies where post-treatment regrowth data were available, a slight delay in regrowth could be observed for the groups where significant tumour size reduction had occurred. Small tumours, below a threshold value ( $\mathrm{T}_{\text {frac }}$, as a proportion of the baseline size) had a reduced growth rate $\left(\lambda_{\text {frac }}\right)$, and a sizedependent effect on growth was found to statistically improve the model fit ( $\Delta \mathrm{OFV}=348.4, \mathrm{X}^{2} \mathrm{p}<0.0001$ ). The reduced growth rate $\lambda_{0 \mathrm{i}}$ is related to the nominal growth rate for that cell line through equation 12 . All anti-tumour activity model parameter estimates are shown in Supplementary Table S4.

Overall, the model presented here adequately describes the population average and individual tumour growth curves with representative comparisons between simulated time-course and observed data, shown in Supplementary Figure S7, and goodness of fit plots are shown in Supplementary Figure S5.

\section{Calculating the level and duration of pMET inhibition required for tumour regression}

Using the model above, the degree and duration of pMET inhibition required to drive anti-tumour activity were determined across the CDX and PDX models investigated. The EBC-1 model had the most extensive dataset available and offered a good way to demonstrate the relationship between inhibition of pMET and anti-tumour activity across the doses and schedules tested. When the time above $90 \%$ pMET inhibition for a weekly cycle is plotted against anti-tumour activity (calculated as the change in growth rate compared to vehicle), maximum anti-tumour activity is observed when pMET inhibition of $>90 \%$ is continuously achieved (Figure 4A). Across the ten CDX and PDX models analysed, all models see $>90 \%$ inhibition which drives anti-tumour activity; however, variable sensitivity was observed in terms of the duration above $90 \%$ required to drive tumour regressions. This can be shown by calculating the time above $90 \%$ inhibition of pMET at a tested dose that delivers tumour stasis (stasis offers a useful endpoint to make a direct comparison) for each CDX and PDX model (Table 2); the most sensitive model (Hs746T) required only approximately $6 \mathrm{~h}$, whilst the least sensitive model (NCI-H441) required continuous coverage. Setting a threshold value, in this case $90 \%$ inhibition, is arbitrary but useful to easily make comparisons across datasets and to enable a baseline understanding of the target modulation requirements without applying more in-depth modelling. However, 
this can be improved upon using the mathematical model presented here with a structure that describes the anti-tumour activity as being dependent on the baseline growth rate of the tumour, and this provides a partial explanation for the level of variation seen across these models. This mathematical relationship can be used to plot the relationship between percentage of pMET inhibition and the ratio of inhibition rate of growth due to savolitinib, over the baseline growth rate of the tumour (Figure 4B). When this ratio is 1 , the rate of inhibition is equal to the tumour growth rate and the tumour is not growing (stasis), whilst values below 1 indicate the tumour is seeing net growth. Values above 1 indicate net tumour shrinkage. This relationship demonstrates that the rate of inhibition of tumour growth initially increases proportional to pMET suppression, but then the inhibitory rate on tumour growth increases disproportionately above $80 \%$ to $90 \%$ pMET suppression. This illustrates the benefit of achieving durable near-maximal inhibition of pMET in driving tumour shrinkage to maximise the tumour inhibitory rate.

\section{Discussion}

Dysregulation of MET signalling is observed in a variety of human cancers with correlations to poor clinical outcomes and drug resistance. Amplification of the MET gene, with consequent constitutive kinase activation has been reported in a number of human primary tumours. Mutations in the MET kinase domain in both sporadic and inherited forms of human renal papillary carcinomas have been previously documented (Olivero et al., 1999). MET exon 14 mutations (MET $\Delta 14$ ) are a recurrent mechanism of MET activation and occurs in around 3-4\% of lung adenocarcinoma (Frampton et al., 2015). Role of MET amplification in resistance to EGFR inhibitors is of significant interest in lung cancer therapy (Lecia V Sequist, 2020). Oncogenic dependency on MET in these various biological contexts and its therapeutic intervention by a plethora of MET-targeted agents as single agents or in combination with novel therapeutics is an area of intense investigation. This warrants a clear understanding of the exposure response relationship of MET-targeted agents in appropriate models. This study showed a robust and consistent relationship between savolitinib PK and pMET inhibition across the models tested.

Several doses/schedules were tested in the EBC-1 lung cancer CDX model to evaluate the extremes of feasible PK profiles across a wide exposure range. More frequent dosing resulted in a stronger response than discontinuous dosing, even when higher doses were administered for the intermittent schedule. This correlates with tumours requiring constant inhibition from savolitinib to drive net tumour shrinkage. This finding is consistent with conclusions derived from a previous study with savolitinib in the Hs746T CDX model (Gu et al., 2019), and studies on crizotinib, where duration of inhibition of pMET was considered the crucial factor for inhibition of tumour growth (Yamazaki et al., 2008). Interestingly, the $\mathrm{EC}_{50}$ for inhibition of pMET of $9.7 \mathrm{ng} / \mathrm{ml}$ derived from Gu et al. was higher than the $0.38 \mathrm{ng} / \mathrm{ml}$ derived from this analysis. Moreover, the degree of pMET inhibition calculated to be required to drive tumour stasis (approximately $10 \%$ ) was lower than our analysis (approximately 80\%) but the plasma concentration required to drive tumour stasis was similar $(1.1 \mathrm{ng} / \mathrm{ml}$ vs. $2.3 \mathrm{ng} / \mathrm{ml})$. The majority of the dataset used by Gu et al. was used in this analysis. We explored these differences and confirmed that in our model, the $\mathrm{EC}_{50}$ for the Hs746T model did not differ statistically from the other CDX and PDX models. Thus, the discrepancy was likely due to differences in modelling approaches applied in the analyses: our dataset was larger, which enabled a more robust assessment of variability to be accounted for in the parameter estimation. Where Gu et al. used a naïve pooling approach, we applied a population approach, which enabled the use of likelihood-based methods to incorporate PK data below the limit of quantification. Additionally, our approach enabled us to estimate the baseline (vehicle) values for pMET as part of the model fitting, instead of correcting for baselines upfront (Gu et al., 2019).

We used a direct response model relating PK to pMET, while Gu et al. used an effect compartment model to explain an observed time-delay between plasma PK and tumour PD. Our analysis did not support the need for a model incorporating any delay. The Gu et al. dataset consisted of PK and PD with the first measurement made at 15 minutes, and the delay calculated to be approximately 1-4 hours. In our datasets, 1 hour was usually the first time-point observed, and therefore less visible; additionally, our data consisted of pMET measurements made after several days' dosing with no time-dependant, or accumulation of effect observed. 
Overall, we felt it was not critical to account for such a time-delay when simulating changes in pMET over time and relating this to tumour growth inhibition over a time period of several weeks. A more pronounced time delay between crizotinib PK and pMET inhibition was evident and thought to be due to rate-limiting pharmacokinetic distribution from plasma to the tumour (Yamazaki, 2013), which would be expected for compounds with a large volume of distribution, such as crizotinib. A PK/PD analysis of tepotinib, together with an analysis in humans, is also available and similarly supports the need for continuous inhibition of pMET (Xiong et al., 2015). The potency of tepotinib to inhibit pMET was 1.7 -fold higher in humans than in mice, and given the precision of the assays available for assessing $\mathrm{EC}_{50}$ and the biological variability observed, this supports a hypothesis of similar translation of inhibitory effect from preclinical to clinical use of savolitinib (Xiong et al., 2015). Taking the $\mathrm{EC}_{90}$ derived from this analysis and correcting it to account for an approximate two-fold difference in plasma protein binding between mouse and human (data unpublished), the estimated plasma concentration required to achieve $90 \%$ pMET inhibition in patients is $7 \mathrm{ng} / \mathrm{ml}$. Data from a Phase I study in patients with advanced solid tumours show that doses above 200-400 mg achieve concentrations in excess of this value at 24 hours (Gan et al., 2019).

Modelling tumour inhibitory effects as a function of tumour growth is a novel approach with the potential to improve translation of tumour growth inhibition from pre-clinical to clinical. We showed that tumour growth inhibition was dependent on the intrinsic growth rate of the tumour, with slower growing tumours being less sensitive to the suppression of pMET signalling. The CDX and PDX models showed that a constant concentration of savolitinib that delivered $80 \%$ pMET suppression could prevent net tumour growth in more sensitive models, whilst the least sensitive models required $>95 \%$ pMET suppression. In practice, savolitinib concentrations in mouse fluctuate by several orders of magnitude over the dosing interval, and at a dose that brings about concentrations that result in continuous cover above $\mathrm{EC}_{90}$, there will be periods when pMET suppression far exceeds $\mathrm{EC}_{90}$. This is desirable to achieve tumour shrinkage and maximise antitumour activity. This model behaviour was consistent with results observed in vivo, where we saw strong tumour shrinkage for the most sensitive models (e.g. Hs746T), whilst tumour stasis was only achievable in the least sensitive (e.g. NCI-H441). Moreover, the inhibitory effect on tumour growth increased exponentially above these threshold values, illustrating the benefit of strongly inhibiting MET continuously for maximum efficacious effect. This supports setting the PK/PD requirement for dosing to achieve $>90 \%$ suppression continuously. Differences in tumour growth rate do not completely explain the heterogeneity in response, observed across the different models, and this is not unexpected as other factors may drive differences in sensitivity to MET inhibition such as additional mutations other than MET .

Our approach to modelling growth inhibition rate as a function of growth rate of the tumour was consistent with the concepts described previously (Norton, 1977). The modification to the Simeoni equation used in this analysis, with linear growth phase and the decreased growth rate for smaller tumours, yields a similar behaviour as the Norton-Simon model for growth over the span of tumour sizes observed.

Due to tumour heterogeneity, anti-cancer therapies show variability in efficacy across a patient population and across disease indications, creating a challenge when trying to model this pre-clinically. Modelling data using multiple mouse models is important to avoid relying on a single, and usually sensitive, model to assess pre-clinically the range in exposure requirements to translate to the clinic. Having PD and efficacy data available from as many models as presented, builds on the work of $\mathrm{Gu}$ et al., where the PD and efficacy effects were explored in one model (the most sensitive in this dataset), and provides further insights on the level of drug exposure required to cover different sensitivities to pathway modulation (Gu et al., 2019). Furthermore, the model presented offers the potential to incorporate tumour dynamics into the translation of anti-tumour effects from a mouse to the patient, by accounting for the differences in the doubling time between a fast-growing mouse tumour and slower-growing human tumours. This could improve the effectiveness of the translation of mouse datasets of tumour efficacy to the patient, and it will be interesting to compare simulations run using this model against patient tumour growth curves in future analyses.

Building a mathematical PK/PD modelling framework is a valuable means to integrate extensive datasets of exposure, pharmacodynamic response and tumour growth to define the target inhibition requirements (and 
potential variability) for optimal efficacy. Savolitinib is being investigated in combination with osimertinib in NSCLC (SAVANNAH: NCT03778229, TATTON: NCT02143466 and ORCHARD: NCT 03944772), and the modelling approach presented here provides a relevant starting point for the combination setting (Ahn et al., 2019; Choueiri et al., 2017; Lecia V Sequist, 2020; Oxnard et al., 2019; Yu et al., 2019). A PK/PD model relating osimertinib exposure to effects on EGF receptor phosphorylation and tumour growth inhibition has been previously published (Yates et al., 2016). Therefore, along with additional data exploring the combination effect in pre-clinical models, this model could be combined with the osimertinib PK/PD model to explore the combination effects of the two agents on PD response and efficacy.

Overall, these results suggest that high and durable levels of MET inhibition are needed for optimal monotherapy in preclinical models. This modelling framework has the potential to translate tumour growth inhibition from mice to humans by adjusting the growth inhibition parameter relative to the intrinsic growth rate of clinical tumours.

\section{Acknowledgments}

The authors acknowledge Lars Lindbom of qPharmetra LLC (Stockholm, Sweden), for assistance with the early modelling work.

This manuscript was funded by AstraZeneca, Cambridge, UK, the manufacturer of savolitinib.

The authors would like to acknowledge Bernadette Tynan, MSc, of Ashfield Healthcare Communications, Macclesfield, UK, part of UDG Healthcare plc, for medical writing support that was funded by AstraZeneca in accordance with Good Publications Practice (GPP3) guidelines (http://www.ismpp.org/gpp3).

\section{References}

Ahn, M.-J., Cantarini, M., Frewer, P., Hawkins, G., Peters, J. A., Howarth, P., . . . Oxnard, G. (2019). SAVANNAH: Phase II trial of osimertinib plus savolitinib in EGFR-mutant, MET-driven advanced NSCLC, following prior osimertinib. Paper presented at the Poster P1.01-134 presented at the IASLC 2019 World Conference on Lung Cancer (WCLC), Barcelona, Spain, 7-10 September 2019.

Albiges, L., Guegan, J., Le Formal, A., Verkarre, V., Rioux-Leclercq, N., Sibony, M., . . Escudier, B. (2014). MET is a potential target across all papillary renal cell carcinomas: result from a large molecular study of pRCC with CGH array and matching gene expression array. Clin Cancer Res, 20 (13), 3411-3421. doi: 10.1158/1078-0432.Ccr-13-2173

Arcila, M. E., Oxnard, G. R., Nafa, K., Riely, G. J., Solomon, S. B., Zakowski, M. F., . . Ladanyi, M. (2011). Rebiopsy of lung cancer patients with acquired resistance to EGFR inhibitors and enhanced detection of the T790M mutation using a locked nucleic acid-based assay. Clin Cancer Res, 17 (5), 1169-1180. doi: 10780432.CCR-10-2277 [pii];10.1158/1078-0432.CCR-10-2277 [doi]

Awad, M. M., Oxnard, G. R., Jackman, D. M., Savukoski, D. O., Hall, D., Shivdasani, P., . . Sholl, L. M. (2016). MET Exon 14 Mutations in Non-Small-Cell Lung Cancer Are Associated With Advanced Age and Stage-Dependent MET Genomic Amplification and c-Met Overexpression.J Clin Oncol, 34 (7), 721-730. doi: $10.1200 /$ jco.2015.63.4600

Bean, J., Brennan, C., Shih, J. Y., Riely, G., Viale, A., Wang, L., . . Pao, W. (2007). MET amplification occurs with or without T790M mutations in EGFR mutant lung tumors with acquired resistance to gefitinib or erlotinib. Proc Natl Acad Sci U S A, 104 (52), 20932-20937. doi: 10.1073/pnas.0710370104

Bladt, F., Faden, B., Friese-Hamim, M., Knuehl, C., Wilm, C., Fittschen, C., . . Blaukat, A. (2013). EMD 1214063 and EMD 1204831 constitute a new class of potent and highly selective c-Met inhibitors. Clin Cancer Res, 19 (11), 2941-2951. doi: 10.1158/1078-0432.CCR-12-3247 
Choueiri, T. K., Jakacki, R., Ghiorghiu, D., Haddad, V., Kohlmann, A., Frigault, M. M., \& Ottesen, L. (2017). 924TiP - Savolitinib versus sunitinib in patients with MET-driven, unresectable and locally advanced or metastatic papillary renal cell carcinoma: SAVOIR, a randomised, phase III trial. Ann Oncol, 28 (suppl_5). doi: $10.1093 /$ annonc/mdx371.078 \%

Cui, Y., Dai, G., Ren, Y., Zhou, F., Fan, S., Sai, Y., . . . Su, W. (2011). Abstract 3612: A novel and selective c-Met inhibitor against subcutaneous xenograft and othotopic brain tumor models. Cancer Res, 71 (8 Supplement), 3612-3612. doi: 10.1158/1538-7445.Am2011-3612

Engelman, J. A., Zejnullahu, K., Mitsudomi, T., Song, Y., Hyland, C., Park, J. O., . . Janne, P. A. (2007). MET amplification leads to gefitinib resistance in lung cancer by activating ERBB3 signaling.Science, 316 (5827), 1039-1043. doi: 10.1126/science.1141478

Falchook, G. S., Hong, D. S., Amin, H. M., Fu, S., Piha-Paul, S. A., Janku, F., . . Kurzrock, R. (2014). Abstract 450PD. First-In-Human Phase I Trial Assessing the Highly Selective C-Met Inhibitor MSC2156119J (EMD 1214063) in Patients with Advanced Solid Tumors. Ann Oncol, 25 (Suppl 4), iv149. doi: 10.1093/annonc/mdu331.10

Frampton, G. M., Ali, S. M., Rosenzweig, M., Chmielecki, J., Lu, X., Bauer, T. M., . . Miller, V. A. (2015). Activation of MET via diverse exon 14 splicing alterations occurs in multiple tumor types and confers clinical sensitivity to MET inhibitors. Cancer Discov, 5 (8), 850-859. doi: 10.1158/2159-8290.Cd-15-0285

Gan, H. K., Millward, M., Hua, Y., Qi, C., Sai, Y., Su, W., . . . Lickliter, J. D. (2019). First-in-Human Phase I Study of the Selective MET Inhibitor, Savolitinib, in Patients with Advanced Solid Tumors: Safety, Pharmacokinetics, and Antitumor Activity. Clin Cancer Res . doi: 10.1158/1078-0432.Ccr-18-1189

Gavine, P. R., Ren, Y., Han, L., Lv, J., Fan, S., Zhang, W., . . Wang, Q. M. (2015). Volitinib, a potent and highly selective c-Met inhibitor, effectively blocks c-Met signaling and growth in c-MET amplified gastric cancer patient-derived tumor xenograft models. Mol Oncol, 9 (1), 323-333. doi: 10.1016/j.molonc.2014.08.015

Gu, Y., Sai, Y., Wang, J., Xia, S., Wang, G., Zhao, Y., . . Su, W. (2013). Abstract 3371. Preclinical disposition and pharmacokinetics of volitinib, a novel selective cMet inhibitor. Cancer Res, 73 (8 Supplement), 3371. doi: 10.1158/1538-7445.AM2013-3371

Gu, Y., Sai, Y., Wang, J., Yu, M., Wang, G., Zhang, L., . . . Su, W. (2019). Preclinical pharmacokinetics, disposition, and translational pharmacokinetic/pharmacodynamic modeling of savolitinib, a novel selective cMet inhibitor. Eur J Pharm Sci, 136 , 104938. doi: 10.1016/j.ejps.2019.05.016

Henry, R. E., Barry, E. R., Castriotta, L., Ladd, B., Markovets, A., Beran, G., . . Schuller, A. G. (2016). Acquired savolitinib resistance in non-small cell lung cancer arises via multiple mechanisms that converge on MET-independent mTOR and MYC activation. Oncotarget, 7 (36), 57651-57670. doi: 10.18632/oncotarget.10859

Jia, H., Dai, G., Weng, J., Zhang, Z., Wang, Q., Zhou, F., . . . Su, W. (2014). Discovery of (S)-1-(1(Imidazo[1,2-a]pyridin-6-yl)ethyl)-6-(1-methyl-1H-pyrazol-4-yl)-1H-[1,2, 3] triazolo[4,5-b]pyrazine (volitinib) as a highly potent and selective mesenchymal-epithelial transition factor (c-Met) inhibitor in clinical development for treatment of cancer. J Med Chem, 57 (18), 7577-7589. doi: 10.1021/jm500510f

Jones, R. D., Grondine, M., Borodovsky, A., Martin, M. S., DuPont, M., D'Cruz, C., . . Ahmed, G. F. (2018). Abstract 4263: A semi-mechanistic pharmacokinetic-pharmacodynamic (PK-PD) model of savolitinib (AZD6094/HMPL-504), a novel MET inhibitor, to explore extent and duration of target inhibition required for optimal efficacy in the EBC-1 mouse xenograft model. Cancer Res, 78 (13 Supplement), 4263-4263. doi: 10.1158/1538-7445.Am2018-4263

Kilkenny, C., Browne, W. J., Cuthill, I. C., Emerson, M., \& Altman, D. G. (2010). Improving bioscience research reporting: The ARRIVE guidelines for reporting animal research. $J$ Pharmacol Pharmacother, 1 (2), 94-99. doi: 10.4103/0976-500x.72351 
Lecia V Sequist, J.-Y. H., Myung-Ju Ahn, Byoung Chul Cho, Helena Yu, Sang-We Kim, James Chih-Hsin Yang, Jong Seok Lee, Wu-Chou Su, Dariusz Kowalski, Sergey Orlov, Mireille Cantarini, Remy B Verheijen, Anders Mellemgaard, Lone Ottesen, Paul Frewer, Xiaoling Ou, Geoffrey Oxnard. (2020). Osimertinib plus savolitinib in patients with EGFR mutation-positive, MET-amplified, non-small-cell lung cancer after progression on EGFR tyrosine kinase inhibitors: interim results from a multicentre, open-label, phase 1b study. The Lancet Oncology, [Epub ahead of print] . doi: 10.1016/S1470-2045(19)30785-5

Lindbom, L., Pihlgren, P., \& Jonsson, E. N. (2005). PsN-Toolkit-a collection of computer intensive statistical methods for non-linear mixed effect modeling using NONMEM. Comput Methods Programs Biomed, 79 (3), 241-257. doi: 10.1016/j.cmpb.2005.04.005

Matsumoto, K., \& Nakamura, T. (1996). Emerging multipotent aspects of hepatocyte growth factor. $J$ Biochem, 119 (4), 591-600.

Norton, L., Simon, R. (1977). Tumor size, sensitivity to therapy, and design of treatment schedules. Cancer Treat Rep, 61 (7), 1307-1317.

Olivero, M., Valente, G., Bardelli, A., Longati, P., Ferrero, N., Cracco, C., . . Di Renzo, M. F. (1999). Novel mutation in the ATP-binding site of the MET oncogene tyrosine kinase in a HPRCC family.Int $J$ Cancer, 82 (5), 640-643. doi: 10.1002/(sici)1097-0215(19990827)82:5<640::aid-ijc4>3.0.co;2-6

Oxnard, G. R., Cantarini, M., Frewer, P., Hawkins, G., Peters, J., Howarth, P., . . Ahn, M.-J. (2019). SAVANNAH: A Phase II trial of osimertinib plus savolitinib for patients (pts) with EGFR-mutant, MET-driven $(\mathrm{MET}+)$, locally advanced or metastatic non-small cell lung cancer (NSCLC), following disease progression on osimertinib. J Clin Oncol, 37 (15_suppl), TPS9119. doi: 10.1200/JCO.2019.37.15_suppl.TPS9119

Raghav, K., Bailey, A. M., Loree, J. M., Kopetz, S., Holla, V., Yap, T. A., . . Hong, D. (2018). Untying the gordion knot of targeting MET in cancer. Cancer Treat Rev, 66 , 95-103. doi: 10.1016/j.ctrv.2018.04.008

Schuller, A. G., Barry, E. R., Jones, R. D., Henry, R. E., Frigault, M. M., Beran, G., . . D’Cruz, C. M. (2015). The MET Inhibitor AZD6094 (Savolitinib, HMPL-504) Induces Regression in Papillary Renal Cell Carcinoma Patient-Derived Xenograft Models. Clin Cancer Res, 21 (12), 2811-2819. doi: 10.1158/10780432.Ccr-14-2685

Sequist, L. V., Waltman, B. A., Dias-Santagata, D., Digumarthy, S., Turke, A. B., Fidias, P., . . Cosper, A. K. (2011). Genotypic and histological evolution of lung cancers acquiring resistance to EGFR inhibitors. Sci Transl Med, 3 . doi: 10.1126/scitranslmed.3002003

Simeoni, M., Magni, P., Cammia, C., De Nicolao, G., Croci, V., Pesenti, E., . . . Rocchetti, M. (2004). Predictive pharmacokinetic-pharmacodynamic modeling of tumor growth kinetics in xenograft models after administration of anticancer agents. Cancer Res, 64 (3), 1094-1101. doi: 10.1158/0008-5472.can-03-2524

Soman, N. R., Correa, P., Ruiz, B. A., \& Wogan, G. N. (1991). The TPR-MET oncogenic rearrangement is present and expressed in human gastric carcinoma and precursor lesions. Proc Natl Acad Sci U S A, 88 (11), 4892-4896. doi: 10.1073/pnas.88.11.4892

Xiong, W., El Bawab, S., Bladt, F., Meyring, M., Klevesath, M., Falchook, G., . . . Girard, P. (2015). Abstract 4510. Model-based phase II dose selection of c-Met inhibitor MSC2156119J. Cancer Res, 75 (15 Supplement), 4510. doi: 10.1158/1538-7445.AM2015-4510

Yamazaki, S. (2013). Translational pharmacokinetic-pharmacodynamic modeling from nonclinical to clinical development: a case study of anticancer drug, crizotinib. AAPS J, 15 (2), 354-366. doi: 10.1208/s12248-0129436-4

Yamazaki, S., Skaptason, J., Romero, D., Lee, J. H., Zou, H. Y., Christensen, J. G., . . Koudriakova, T. (2008). Pharmacokinetic-pharmacodynamic modeling of biomarker response and tumor growth inhibition to an orally available cMet kinase inhibitor in human tumor xenograft mouse models. Drug Metab Dispos, 36 (7), 1267-1274. doi: 10.1124/dmd.107.019711 
Yates, J. W. T., Ashton, S., Cross, D., Mellor, M. J., Powell, S. J., \& Ballard, P. (2016). Irreversible Inhibition of EGFR: Modeling the Combined Pharmacokinetic-Pharmacodynamic Relationship of Osimertinib and Its Active Metabolite AZ5104. 15 (10), 2378-2387. doi: 10.1158/1535-7163.MCT-16-0142 \%J Molecular Cancer Therapeutics

Yu, H., Ahn, M.-J., Kim, S.-W., Cho, B. C., Sequist, L., Orlov, S., . . Han, J.-Y. (2019). Abstract CT032: TATTON Phase Ib expansion cohort: Osimertinib plus savolitinib for patients (pts) with EGFR-mutant, MET-amplified NSCLC after progression on prior first/second-generation epidermal growth factor receptor (EGFR) tyrosine kinase inhibitor (TKI).Cancer Res, 79 (13 Supplement), CT032-CT032. doi: 10.1158/15387445.SABCS18-CT032 \%J Cancer Research

Tables

Table 1. Model parameter estimates for the mouse PK model.

\begin{tabular}{lllll}
\hline Parameter & Unit & Description & Estimate & $95 \%$ CI \\
$\mathrm{V}_{\max }$ & $\mathrm{mg} / \mathrm{h} / \mathrm{kg}$ & Maximum elimination rate & 2.41 & $2.36-3.61$ \\
$\mathrm{~V}_{\mathrm{c}}$ & $\mathrm{L} / \mathrm{kg}$ & Central volume & 0.39 & $0.277-0.446$ \\
$\mathrm{k}_{\mathrm{a}}$ & $/ \mathrm{h}$ & Absorption rate constant & 5.07 & $3.17-5.98$ \\
$\mathrm{~V}_{\mathrm{p}}$ & $\mathrm{L} / \mathrm{kg}$ & Peripheral volume & 0.89 & $0.602-0.934$ \\
$\mathrm{Q}$ & $\mathrm{L} / \mathrm{h} / \mathrm{kg}$ & Inter-compartment clearance & 0.65 & $0.465-0.765$ \\
$\mathrm{~F}$ & $\%$ & Bioavailability & 28.0 & $25.9-35.0$ \\
$\mathrm{KM}$ & $\mu \mathrm{g} / \mathrm{kg}$ & Amount of half $\mathrm{V}_{\max }$ & 616 & $478-972$ \\
Proportional error & $\%$ & Proportional residual error & 45 & $39-50$ \\
Additive error & $\mathrm{ng} / \mathrm{ml}$ & Additive residual error & 4.7 & $1.4-2.8$ \\
$\mathrm{IIV}\left(\mathrm{V}_{\max }\right)$ & $\%$ & Inter-individual variability $\mathrm{V}_{\max }$ & 28.2 & $24.4-32.2$ \\
\hline
\end{tabular}

Table 2. Simulated duration of pMET inhibition at savolitinib doses delivering near stasis in xenografted tumour growth inhibition studies.

\begin{tabular}{|c|c|c|c|c|c|c|}
\hline Tumour & Indication & $\begin{array}{l}\text { MET } \\
\text { status } \\
\text { (copy } \\
\text { number) }\end{array}$ & $\begin{array}{l}\text { Other } \\
\text { known } \\
\text { mutations }\end{array}$ & $\begin{array}{l}\text { Once-daily } \\
\text { dose that } \\
\text { delivers as } \\
\text { near to } \\
\text { tumour } \\
\text { growth } \\
\text { stasis as } \\
\text { tested [mg } \\
(\mathrm{TG})]\end{array}$ & $\begin{array}{l}\text { Time } \\
\text { above } \\
\text { pMET } \\
\text { EC90 } \\
\text { required to } \\
\text { deliver } \\
\text { tumour } \\
\text { stasis } \\
\text { (hours) }\end{array}$ & Reference \\
\hline EBC-1 & $\begin{array}{l}\text { Squamous } \\
\text { NSCLC }\end{array}$ & CN gain (14) & P53, ATM & $30(76-94 \%)$ & 12 & $\begin{array}{l}\text { (Jones et al., } \\
\text { 2018) This } \\
\text { study }\end{array}$ \\
\hline NCI-H1993 & NSCLC & CN gain (14) & P53, STK11 & $>30(65 \%)$ & 14.5 & $\begin{array}{l}\text { (Henry et al., } \\
2016 \text { ) Data on } \\
\text { file }\end{array}$ \\
\hline NCI-H441 & NSCLC & $\mathrm{CN}$ gain $(3)$ & P53, KRAS & $100(89 \%)$ & 23 & Data on file \\
\hline $\begin{array}{l}\text { HLXF- } \\
\text { 036LN }\end{array}$ & NSCLC & $\begin{array}{l}\text { CN gain } \\
(3.7)\end{array}$ & P53 & $50(-28 \%)$ & 18 & $\begin{array}{l}\text { (Henry et } \\
\text { al., 2016) }\end{array}$ \\
\hline $\mathrm{RCC} 43 \mathrm{~b}$ & PRCC & CN gain (8) & BRCA1 m & $10(100 \%)$ & 10 & $\begin{array}{l}\text { (Schuller et } \\
\text { al., 2015) }\end{array}$ \\
\hline $\mathrm{RCC} 47$ & PRCC & $\mathrm{CN}$ gain $(9)$ & - & $2.5(89 \%)$ & 6 & $\begin{array}{l}\text { (Schuller et } \\
\text { al., 2015) }\end{array}$ \\
\hline
\end{tabular}




\begin{tabular}{|c|c|c|c|c|c|c|}
\hline SNU-5 & Gastric & $\begin{array}{l}\text { CN gain } \\
(17.9)\end{array}$ & $\begin{array}{l}\text { P53, IDH2, } \\
\text { CDH1 }\end{array}$ & $5(97 \%)$ & 8 & $\begin{array}{l}\text { (Cui et al., } \\
2011 \text { ) }\end{array}$ \\
\hline $\mathrm{Hs} 746 \mathrm{~T}$ & Gastric & $\begin{array}{l}\text { CN gain } \\
(21.3)\end{array}$ & $\begin{array}{l}\text { P53, BRCA, } \\
\text { APC }\end{array}$ & $2.5(97 \%)$ & 6 & $\begin{array}{l}\text { (Gavine et } \\
\text { al., 2015) }\end{array}$ \\
\hline MKN-45 & Gastric & $\begin{array}{l}\mathrm{CN} \text { gain } \\
(21)\end{array}$ & $\mathrm{p} 53$ & $25(100 \%)$ & 11 & This study \\
\hline NCI-H820 & NSCLC & $\mathrm{CN}$ gain (6) & P53, STK11 & $>30(73 \%)$ & 12 & Data on file \\
\hline
\end{tabular}

\section{Figure legends}

Figure 1 Mouse exposure (predicted savolitinib plasma concentration) versus pMET inhibition for the complete set of CDX and PDX models used for this analysis. Line fit using an $\mathrm{E}_{\max }$ model (shade area is 95\% CI). Across these studies, a mixture of tumour samples was analysed for pMET by MSD and WB.

Figure 2 Plots comparing model simulations of pMET inhibition time-course with observed data following a single administration of savolitinib at various doses. A : MKN-45; B : EBC-1

A

B

Figure 3 Tumour growth inhibition with savolitinib. Aand B : EBC-1 cell line models; C : MKN-45 cell line models.

Figure 4 Predicted relationship between pMET inhibition with savolitinib and anti-tumour activity as ratio of kill/growth. A value of 1 for anti-tumour activity equates to tumour stasis (no net growth), whilst values greater than 1 equate to tumour regressions. The modelled relationships are plotted per CDX/PDX or group of models. A:Time spent with pMET inhibition $>90 \%$ and anti-tumour activity; B: Percentage change from baseline in pMET inhibition and anti-tumour activity; the table provides parameter estimates for slope and $\gamma$.

A

B 


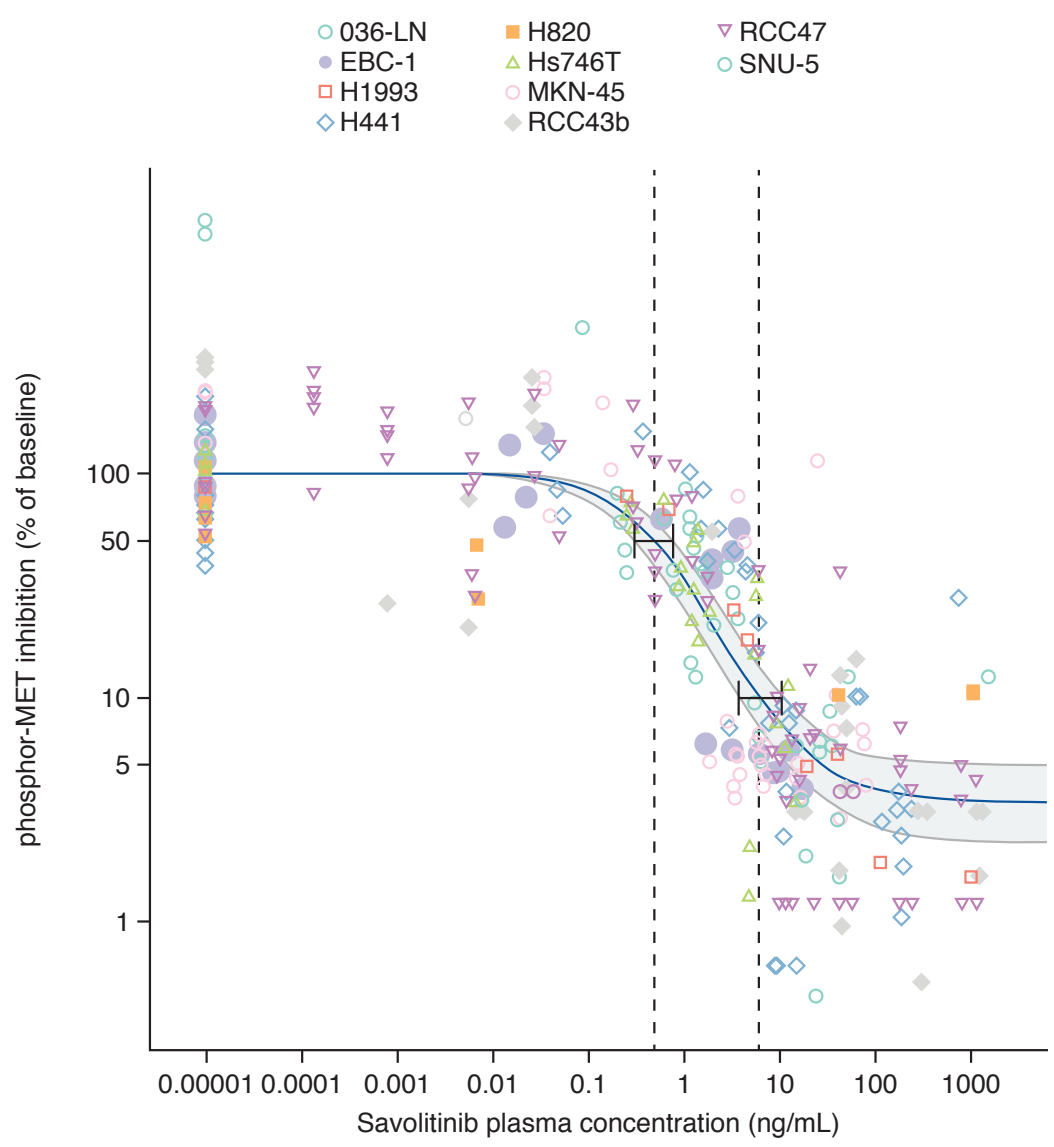




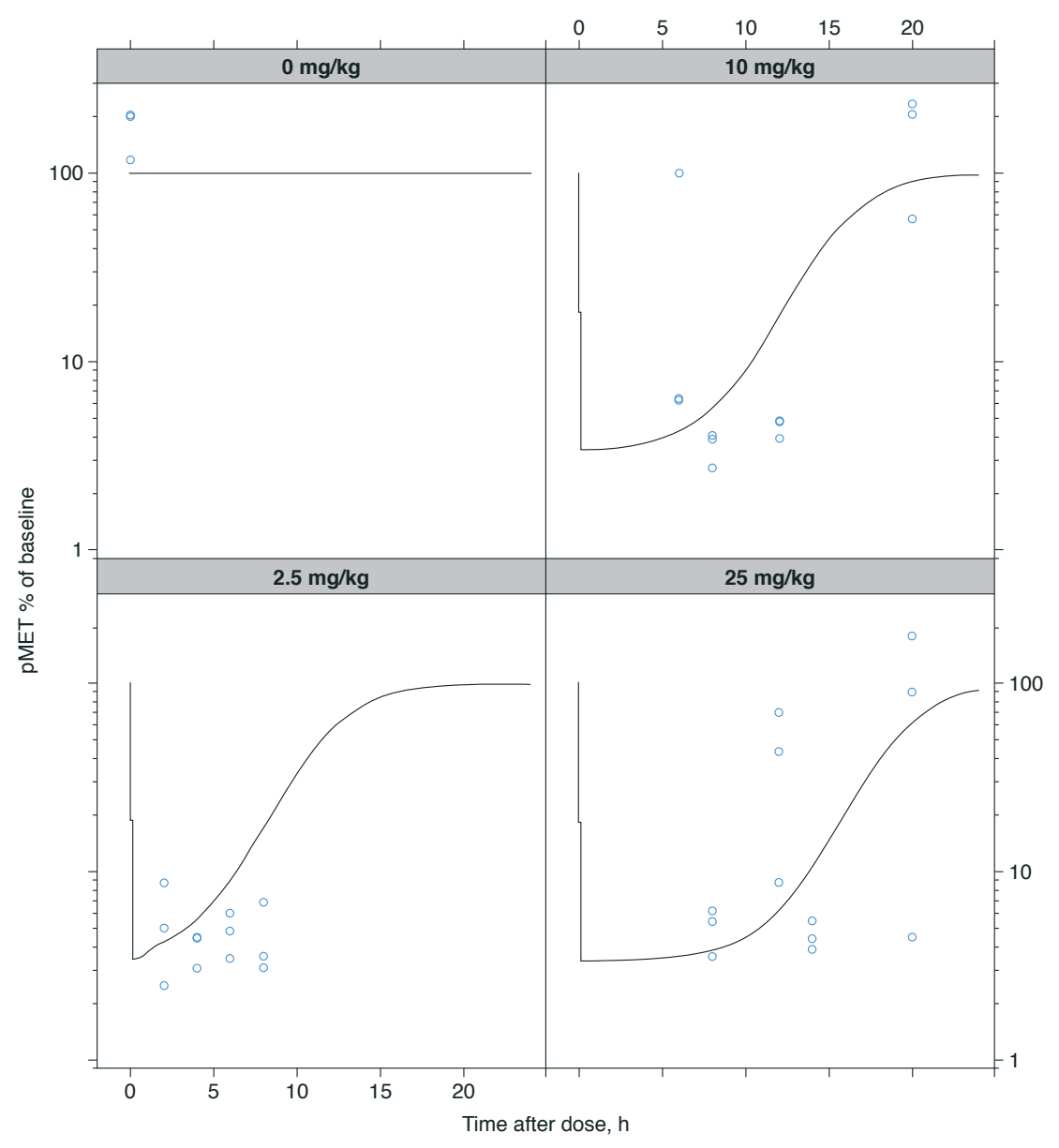




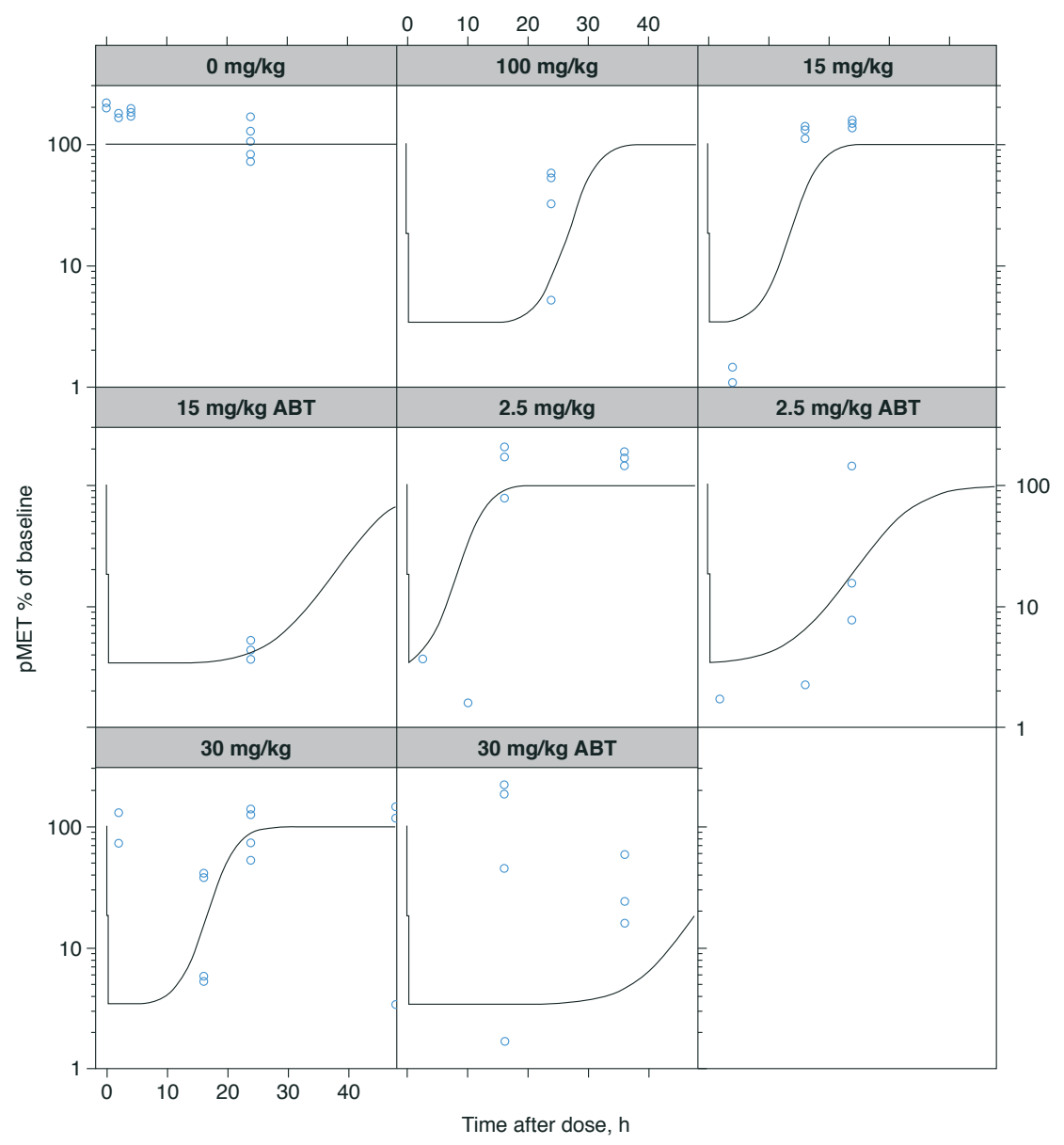

A.

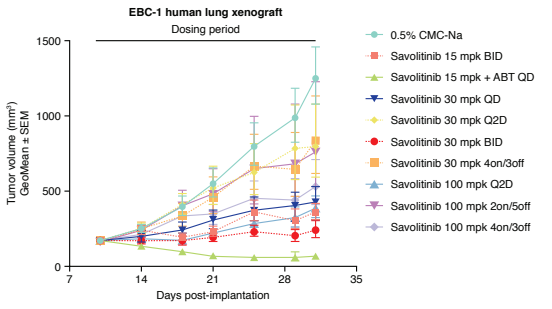

B.

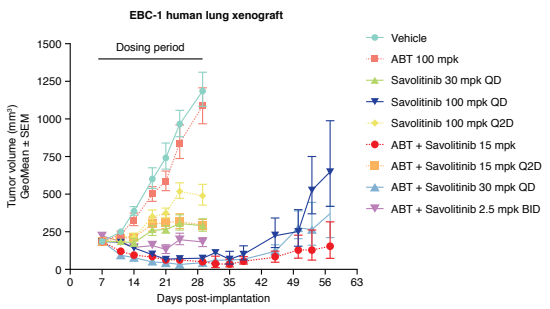

c.

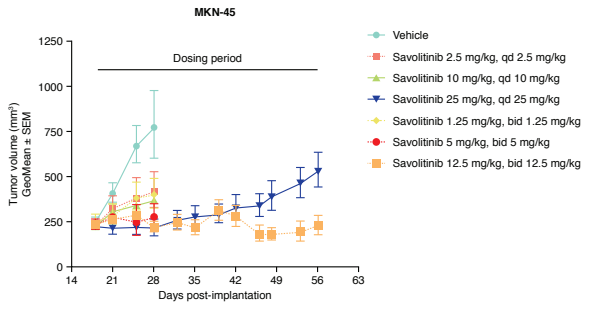




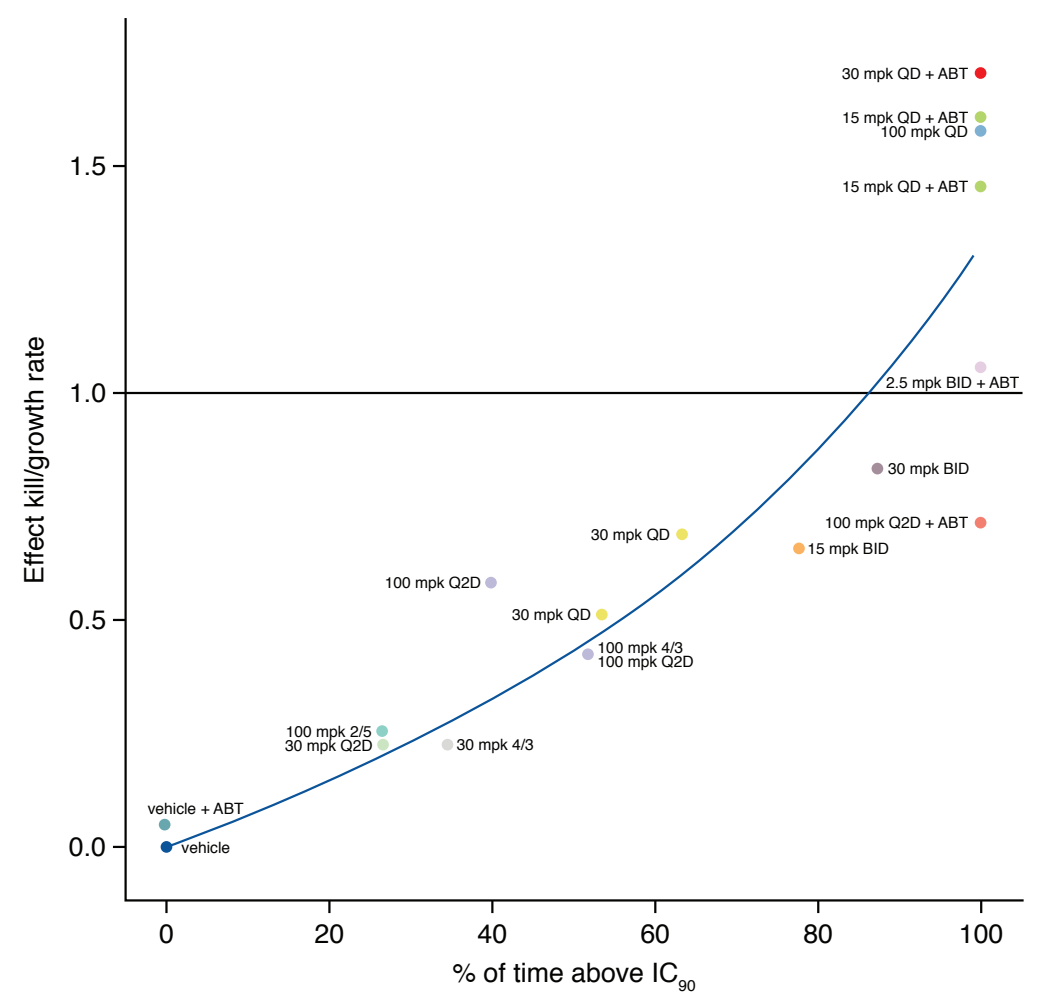




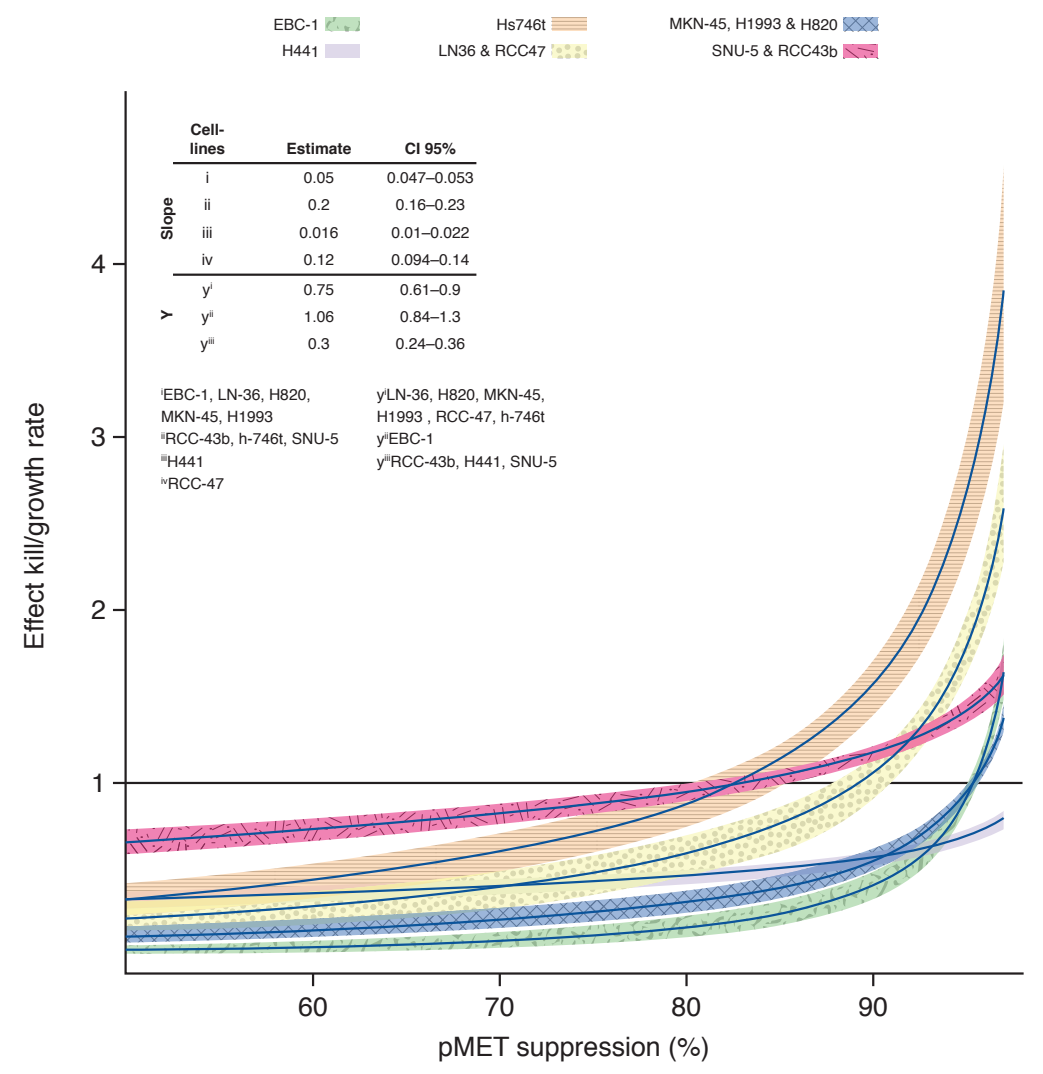

